\begin{tabular}{l} 
Sharif University of Technology \\
Scientia Iranica \\
SCIENTIA \\
IRANICA \\
Transtions A: Civil Engineering \\
\hline
\end{tabular}

\title{
Uncertainty analysis of open channel flow: Stochastic approach to advection-diffusion equation
}

\author{
H. Khorshidi*, N. Talebbeydokhti and Gh. Rakhshandehroo \\ Department of Civil and Environmental Engineering, Shiraz University, Shiraz, Iran.
}

Received 9 October 2015; received in revised form 18 April 2016; accepted 18 June 2016

\section{KEYWORDS}

Advection-diffusion equation;

Karhunen-Loeve expansion;

Monte Carlo

simulation;

Open channel flow;

Stochastic solution.

\begin{abstract}
In fluid mechanics applications, transport occurs through the combination of advection and diffusion. This paper presents a stochastic approach to describe uncertainty and its propagation based on Advection-Diffusion Equation. To assess the uncertainty in initial water depth, random initial condition is imposed on the framework of 1D open cannel flow. Karhunen-Loeve Expansion is adopted to decompose the uncertain parameter in terms of infinite series containing a set of orthogonal Gaussian random variables. Eigenstructures of covariance function associated with the random parameter, which play a key role in computing coefficients of the series, are extracted from Fredhulm's equation. The flow depth is also represented as an infinite series of its moments, obtained via polynomial expansion decomposition in terms of the products of random variables. Coefficients of these series are obtained by a set of recursive equations derived from the ADE. Results highlight the effect of various statistical properties of initial water depth. The mean value and variance for the flow depth are compared with Monte Carlo Simulation as a reliable stochastic approach. It is found that when higher-order approximations are used, KLE results would be as accurate as the results of MCS, however, with much less computational time and effort.
\end{abstract}

(C) 2017 Sharif University of Technology. All rights reserved.

\section{Introduction}

Given the heterogeneous nature of many fluid flows and difficulties associated with understanding this heterogeneity accurately, flow characteristics are often treated as random functions, leading to governing equations of stochastic types. Hence, it is no surprise that statistical estimation of such stochastic processes has received considerable attention as an active field in realworld simulations. Although there has been continuous effort to develop stochastic models in various fields of

*. Corresponding author. Fax: +98 7136473161

Email addresses: khorshidi@shirazu.ac.ir (H. Khorshidi);

taleb@shirazu.ac.ir (N. Talebbeydokhti);

rakhshan@shirazu.ac.ir (G.Rakhshandehroo) fluid flows, their application in open channel flows has received less attention.

Advection-Diffusion Equation (ADE) arises usually in transport modeling fields of applications and in any disturbance analysis of surface water flows. In such flows, field parameters are often influenced by uncertainty due to the lack of understanding of natural open channel properties including roughness coefficient, bed slope, and initial or boundary conditions.

A conventional method to solve Partial Differential Equations (PDEs) stochastically is Polynomial Chaos Expansion (PCE). It was put forward by Ghanem and Spanos [1], with application to transport in heterogeneous media $[2,3]$ and diffusion problems [4]. PCE is applied to model the uncertainty propagation from the beginning of a waterhammer with random system parameters and internal boundary conditions [5]. This technique includes representing 
the random variables in terms of polynomial chaos basis and deriving appropriate discretized equations for the expansion coefficients via Galerkin technique. PCE allows for high-order approximation of random variables and possesses fast convergence under certain conditions. However, the deterministic coefficients of PCE are governed by a set of coupled equations, which are difficult to solve when the number of coefficients is large. PCE is based on the expansion of variables by products of polynomial coefficients and orthogonal chaos bases. It is needed to treat a system of equations numerically. PCE applications to stochastic shallow water flows were reported by Ge et al. [6] and Liu [7]. A comprehensive review of PCE approach is presented by Debusschere et al. [8].

Karhunen-Loeve Expansion (KLE) is a flexible approach to solve PDEs stochastically, leading to high-order moments with relatively small computational efforts. PCE $[9,10]$, probabilistic collocation method [11,12], and KLE [9,13,14] have been utilized to illustrate random processes in porous media. This method is applied to decompose the solution to Boussinesq equations for the velocity, density, and pressure fields [15].

KLE approach has proved to be efficient for uncertainty analysis in groundwater hydraulics $[9,13,16]$. Contrary to PCE, the coefficients associated with KLE appear in uncoupled equations, from which the required statistical moments can be extracted. However, this method has received little attention in open channel applications.

A reliable tool usually used as a reference for solving stochastic PDEs is Monte Carlo Simulation (MCS), which consists of three steps; 1) generating several realizations of the uncertain parameter based on its distribution function, 2) solving the governing equation by means of an appropriate deterministic scheme for the generated parameter, and 3) taking statistical moments on the entire realizations obtained in previous steps. MCS is simple to implement; however, it requires considerable computational effort due to large number of realizations needed. Application of MCS in open channel flow has been reported by Gates and Alzahrani [17,18] for Colombia River in US. The uncertainties in geometrical properties and bed slope are investigated via their distribution functions and, consequently, statistical moments are evaluated for the flow field. A virtual sampling MCS is proposed to address uncertainty quantification in flood modeling on a real test case for Tous dam break in Spain [19]. Dutykh et al. [20] adopted MCS to quantify the effect of bottom roughness on maximum run-up height by resorting to nonlinear shallow water equations. Multilevel MCS is applied to uncertainty quantification for porous media flow [21].

In the present work, KLE approach is applied to
1-D Advection-Diffusion equation considering uncertainty in the initial condition for a synthetic case in open channel flow. Consequently, flow field parameter has appeared as a random variable, too. Validity of the proposed model has been ensured through comparing it with MCS results for various spatial variability and correlation lengths.

\section{ADE governing equation}

On the Advection Diffusion equation method for water wave propagation, transport occurs through the combination of advection and diffusion. ADE is derived mostly from the incorporation of advection into diffusion equation, and could be utilized in different geometries and conditions. The derivation of the ADE relies on the principle of superposition: Advection and diffusion can be added together if they are linearly independent; the only way they can be dependent is when one process feeds back into the other. One-dimensional ADE could be written for an incompressible fluid as:

$$
\frac{\partial H}{\partial t}+U \frac{\partial H}{\partial x}-D \frac{\partial^{2} H}{\partial x^{2}}=0
$$

in which $H(x, t)$ is flow depth, $U$ is flow velocity (or advective velocity), and $D$ is diffusive coefficient (or diffusivity), subject to the initial and boundary conditions given by:

$$
\begin{aligned}
& H(x, 0)=h_{0}+a \operatorname{sech}^{2}\left[\sqrt{\frac{3 a}{4 h_{0}^{3}}}\left(x-\frac{L}{2}\right)\right], \quad x \in \underset{(2)}{D,} \\
& H(0, t)=H(L, t)=h_{0}, \quad t>0,
\end{aligned}
$$

where $L$ is channel length, $H(x, 0)$ or $h(x)$ is initial water depth (treated hereafter as a random variable), $h_{0}$ is undisturbed uniform water depth, $a$ is initial wave height, and $D$ is spatial domain in $x$ direction. The initial condition corresponds to a first-order solitary wave propagating in the positive $x$-direction [22]. The random nature of $h(x)$ converts deterministic equations (Eqs. (1)-(3)) into stochastic ones, the solution to which is sought in the form of statistical moments. The length of the channel is assumed sufficiently large compared to the characteristic length of solitary wave [23]. This justifies the validity of Eq. (3), implying that the boundary values remain unaffected by the initial wave form.

\section{KLE stochastic representation of $\mathrm{ADE}$}

In KLE approach, initial water depth, $h(x)$, is considered a random variable due to many factors including the uncertainty inherent in measurements. It may be decomposed to the mean term, $\langle h>$, and the 
fluctuation term, $h^{\prime}$. KLE expresses $h^{\prime}(x)$ in terms of eigenstructure for covariance function, $C_{h}\left(x_{1}, x_{2}\right)$, of the random field as follows [1]:

$$
h^{\prime}(x, \omega)=\sum_{n=1}^{\infty} \xi_{n}(\omega) \sqrt{\lambda_{n}} f_{n}(x),
$$

where $x$ and $\omega$ are indices of real and probability spaces, respectively; $\xi_{n}(\omega)$ is an orthogonal Gaussian random variable with zero mean; and $\lambda_{n}$ and $f_{n}(x)$ are eigenvalue and eigenfunction associated with the given covariance function, respectively. With a covariance function for the exponential distribution as:

$$
C_{h}\left(x_{1}, x_{2}\right) \sigma_{h}^{2} \exp \left(-\frac{\left|x_{1}-x_{2}\right|}{\eta}\right),
$$

the eigenstructures are obtained analytically from Fredholm's equation [13] as:

$$
\begin{aligned}
& \lambda_{n}=\frac{2 \eta \sigma_{h}^{2}}{\eta^{2} w_{n}^{2}}, \\
& f_{n}(x)=\frac{\left[\eta w_{n} \cos \left(w_{n} x\right)+\sin \left(w_{n} x\right)\right]}{\sqrt{0.5 L\left(\eta^{2} w_{n}^{2}+1\right)+\eta}},
\end{aligned}
$$

where $\sigma_{h}^{2}$ and $\eta$ are variance and correlation length of the random variable $h(x, \omega)$, respectively. It is worth mentioning that a similar problem has been treated by Zhang and $\mathrm{Lu}$ [13] when modeling groundwater flow in a random porous medium. In the above expression, $w_{n}$ refers to positive roots of the characteristic equation:

$$
\left(\eta^{2} w^{2}-1\right) \sin (w L)=2 \eta w \cos (w L) .
$$

For notational convenience, the function $\sqrt{\lambda_{n}} f_{n}(x)$ is replaced with $f_{n}^{*}(x)$ hereafter.

\section{Moment equations in KLE}

As mentioned, $h(x)$ is considered as a random variable and other terms as deterministic ones. KLE, as a perturbative expansion technique, expands the dependent variable $H(x, t)$ by the following series:

$$
H(x, t)=H^{(0)}+H^{(1)}+H^{(2)}+\ldots
$$

Substituting the above expansion and $h(x)=<h>$ $+h^{\prime}$ in Eqs. (1)-(3) and considering only the zero-order terms, the governing equation and related conditions will take the form:

$$
\begin{aligned}
& \frac{\partial H^{(0)}}{\partial t}+U \frac{\partial H^{(0)}}{\partial x}-D \frac{\partial^{2} H^{(0)}}{\partial x^{2}}=0 \\
& H^{(0)}(x, 0)=h_{0}+\bar{a} \operatorname{sech}^{2}\left[\sqrt{\frac{3 a}{4 h_{0}^{3}}}\left(x-\frac{L}{2}\right)\right], x \in D \\
& H^{(0)}(0, t)=H^{(0)}(L, t)=h_{0}, \quad t>0
\end{aligned}
$$

in which $\bar{a}$ is mean value of $a$. The above equation may be solved for $H^{(0)}$ in a deterministic manner (Appendix A). Similarly, one may obtain the following expression for any higher order term $(m)$ [13]:

$$
\begin{aligned}
U \frac{\partial}{\partial x}\left(H^{(m)}\right) & -D \frac{\partial^{2}}{\partial x^{2}}\left(H^{(m)}\right) \\
& +\sum_{k=0}^{m} \frac{(-1)^{k}}{k !}\left[h^{\prime}(x)\right]^{k} \frac{\partial H^{(m-k)}}{\partial t}=0, \\
H^{(m)}(x, 0) & =0, \quad x \in D \\
H^{(m)}(0, t) & =H^{(m)}(L, t)=0 .
\end{aligned}
$$

Various components of Eq. (7) can now be expanded by suitable polynomial expansions in terms of the

\begin{tabular}{|c|c|c|}
\hline & Term & Governing equation \\
\hline First term & $H^{(1)}(x, t)=\sum_{i=1}^{\infty} \xi_{i} H_{i}^{(1)}(x, t)$ & $\frac{\partial H_{i}^{(1)}}{\partial t}-f_{n}^{*}(x) \frac{\partial H^{(0)}}{\partial t}+U \frac{\partial}{\partial x}\left(H_{i}^{(1)}\right)-D \frac{\partial^{2}}{\partial x^{2}}\left(H_{i}^{(1)}\right)=0$ \\
\hline Second term & $H^{(2)}(x, t)=\sum_{i, j=1}^{\infty} \xi_{i} \xi_{j} H_{i j}^{(2)}(x, t)$ & $\begin{aligned} \frac{\partial H_{i j}^{(2)}}{\partial t}- & \frac{1}{2} f_{i}^{*}(x) \frac{\partial H_{j}^{(1)}}{\partial t}-\frac{1}{2} f_{j}^{*}(x) \frac{\partial H_{i}^{(1)}}{\partial t}+\frac{1}{2} f_{i}^{*}(x) f_{j}^{*}(x) \frac{\partial H^{(0)}}{\partial t} \\
& +U \frac{\partial H_{i j}^{(2)}}{\partial x}-D \frac{\partial^{2} H_{i j}^{(2)}}{\partial x^{2}}=0\end{aligned}$ \\
\hline Third term & $\begin{aligned} H^{(3)}(x, t)= & \sum_{n=1}^{\infty} \xi_{n} H_{n}^{(3)}(x, t) \\
& +\sum_{i, j, k=1}^{\infty} \xi_{i} \xi_{j} \xi_{k} H_{i j k}^{(3)}(x, t)\end{aligned}$ & $\begin{aligned} \frac{\partial H_{i j k}^{(3)}}{\partial t} & -\frac{1}{3} \sum_{P_{i j k}} f_{i}^{*}(x) \frac{\partial H_{j k}^{(2)}}{\partial t}+\frac{1}{6} \sum_{P_{i j k}} f_{i}^{*}(x) f_{j}^{*}(x) \frac{\partial H_{k}^{(1)}}{\partial t} \\
& -\frac{1}{6} \sum_{P_{i j k}} f_{i}^{*}(x) f_{j}^{*}(x) f_{k}^{*}(x) \frac{\partial H^{(0)}}{\partial t}+U \frac{\partial H_{i j k}^{(3)}}{\partial x}-D \frac{\partial^{2} H_{i j k}^{(3)}}{\partial x^{2}}=0\end{aligned}$ \\
\hline
\end{tabular}
orthogonal Gaussian random variable $\xi$ as illustrated in Table 1. where $H_{i}^{(1)}, H_{i j}^{(2)}$, and $H_{i j k}^{(3)}$ (for $i, j, k=$ $1,2, \ldots)$ are deterministic coefficients obtained from the associated governing equation, numerically. Note that the above governing equations are derived via substituting the expansions of $h^{\prime}(x)$ and $H^{(m)}(x, t)$ with $m=1,2,3$ in Eqs. (11)-(13) and simplifying the resulting expressions in view of orthogonality of the random variable, $\xi$. The equations listed in Table 1 are treated recursively in a numerical manner (Appendix).

Table 1. Expansions for $H^{(1)}, H^{(2)}$, and $H^{(3)}$ in terms of the orthogonal Gaussian random variable $\xi$, and the governing equation for any term. 
Table 2. Statistical moments of the flow depth.

\begin{tabular}{|c|c|}
\hline Flow depth & $H(x, t) \approx \sum_{i=0}^{3} H^{(i)}(x, t)$ \\
\hline Mean value & $\langle H(x, t)\rangle \approx \sum_{i=0}^{3}\left\langle H^{(i)}(x, t)\right\rangle=H^{(0)}(x, t)+\sum_{i=1}^{\infty} H_{i i}^{(2)}(x, t)$ \\
\hline Perturbation term & $H^{\prime}(x, t)=H(x, t)-\langle H(x, t)\rangle \approx \sum_{i=1}^{3} H^{(i)}(x, t)-\sum_{i=1}^{\infty} H_{i i}^{(2)}(x, t)$ \\
\hline $\begin{array}{l}\text { Cross-covariance between initial } \\
\text { water depth and flow depth }\end{array}$ & $\begin{aligned} C_{h H}(x ; y ; \tau)= & \sqrt{\lambda_{n}} f_{n}(x) H^{\prime}(x, \omega)=f_{n}^{*}(x) H^{\prime}(x, \omega)=\sum_{n=1}^{\infty} f_{n}^{*}(x) H_{n}^{(1)}(y, \tau) \\
& +3 \sum_{i, j=1}^{\infty} f_{i}^{*}(x) H_{i j j}^{(3)}(y, \tau)\end{aligned}$ \\
\hline Flow depth covariance & $\begin{aligned} C_{H}(x, t ; y, \tau)= & \sum_{i=1}^{\infty} H_{i}^{(1)}(x, t) H_{i}^{(1)}(y, \tau)+2 \sum_{i, j=1}^{\infty} H_{i j}^{(2)}(x, t) H_{i j}^{(2)}(y, \tau) \\
& +3 \sum_{i, j=1}^{\infty} H_{i}^{(1)}(x, t) H_{i j j}^{(3)}(y, \tau)+3 \sum_{i, j=1}^{\infty} H_{i}^{(1)}(y, \tau) H_{i j j}^{(3)}(x, t)\end{aligned}$ \\
\hline Flow depth variance & $\sigma_{H}^{2}(x, t)=\sum_{i=1}^{\infty}\left[H_{i}^{(1)}(x, t)\right]^{2}+2 \sum_{i, j=1}^{\infty}\left[H_{i j}^{(2)}(x, t)\right]^{2}+6 \sum_{i, j=1}^{\infty} H_{i}^{(1)}(x, t) H_{i j j}^{(3)}(x, t)$ \\
\hline
\end{tabular}

Index $\sum_{P_{i j k}}($.$) is found by a substitution manner, i.e.:$

$$
\begin{aligned}
\sum_{P_{i j k}} \nabla f_{i}^{*} \cdot \nabla H_{j k}^{(2)}= & \nabla f_{i}^{*} \cdot \nabla H_{j k}^{(2)}+\nabla f_{j}^{*} \cdot \nabla H_{i k}^{(2)} \\
& +\nabla f_{k}^{*} \cdot \nabla H_{i j}^{(2)}
\end{aligned}
$$

For the trivial solutions to exist, $H^{(3)}(x, t)$ should be expanded in terms of $\xi_{n}$ and $\xi_{i} \xi_{j} \xi_{k}$ simultaneously [13]. Manipulating the third-order approximation of $H(x, t)$ (Eq. (7)) mathematically, one may compute higher moments of the flow depth as shown in Table 2. It is important to note that the same approach is chosen to solve the governing equation of $H^{(0)}$ to $H_{i j k}^{(3)}$ because of the diversity in homogeneity property. Despite the existence of analytical solution to Eqs. (8)-(10) (i.e., convolution integral), QUICKEST approach (as a finite difference method expressed in Appendix) is utilized to treat all the governing equations to have the same solution process.

\section{Hypothetical test problem}

KLE approach is applied to a hypothetical channel (Figure 1) to compute higher-order flow depth moments, and verified by comparing its results with those of MCS. A hypothetical channel of length $L=100 \mathrm{~m}$ is considered with a first-order solitary wave with maximum height of $a$ within normal distribution and mean value of $\bar{a}=0.05 \mathrm{~m}$ centered at $x=L / 2$. Moreover, the water depth is kept constant at $h_{0}=1 \mathrm{~m}$ over the channel ends, advective velocity $U=2.5 \mathrm{~m} / \mathrm{s}$, and diffusion coefficient $D=1 \mathrm{~m}^{2} / \mathrm{s}$. Schematic of the initial condition over the channel and wave propagation sketch at different times are shown in Figure 1. Effects of different correlation lengths, $\eta_{h}$, and various degrees of spatial variability, $\sigma_{h}^{2}$, on flow depth variance, $\sigma_{H}^{2}$, have been investigated. MCS is examined for about 1000 realizations and the moments

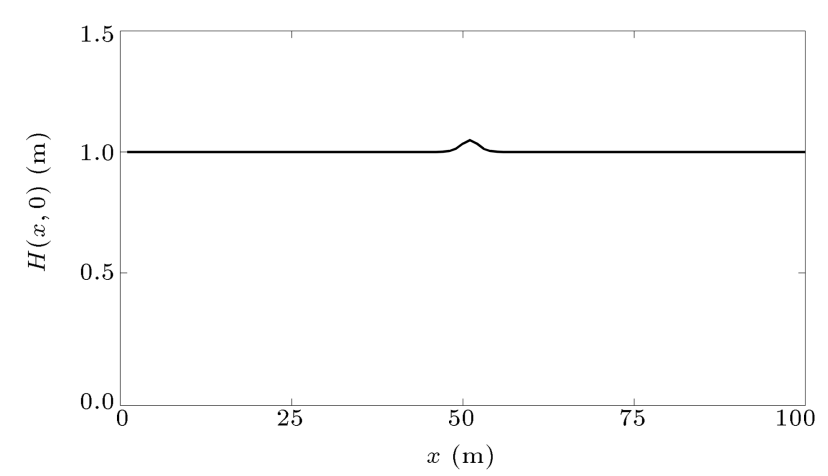

(a)

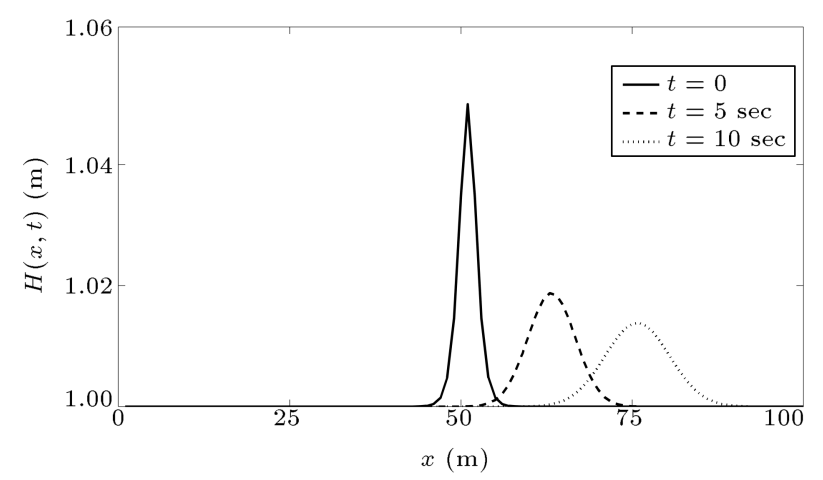

(b)

Figure 1. (a) Schematic of the initial condition. (b) Wave propagation sketch at different times.

of flow depth are computed for different correlation lengths and variances of the input random variable.

\section{Results and discussion}

A sufficient number of terms to be incorporated in $H(x, t)$ and, subsequently, in expansions of $H^{(m)}$ are investigated. Furthermore, effects of correlation length and spatial variability of random variable $h(x)$ on variance of the random function $\sigma_{H}^{2}$ have been discussed. 


\subsection{Number of terms to be incorporated in $\boldsymbol{H}(\boldsymbol{x . t})$}

KLE approach is applied to compute mean flow depth profile at different times, and compared with MCS ones as shown in Figure 2. Incorporation of the first two terms in Eq. (7) leads the results to a good agreement with the results of MCS (Figure 2(a)). Incorporating two more terms, i.e., $H^{(2)}$ and $H^{(3)}$, only slightly improves $H(x, t)$ (Figure 2(b) and (c)). Indeed, numerical values of subsequent terms decrease by one order of magnitude. For example, $H^{(1)}(x, t)$ (with a certain number of terms considered in its expansion as illustrated in the next section) takes the value of $0.01 \mathrm{~m}$; however, the value of $0.001 \mathrm{~m}$ is obtained in estimation of $H^{(2)}(x, t)$ with sufficient

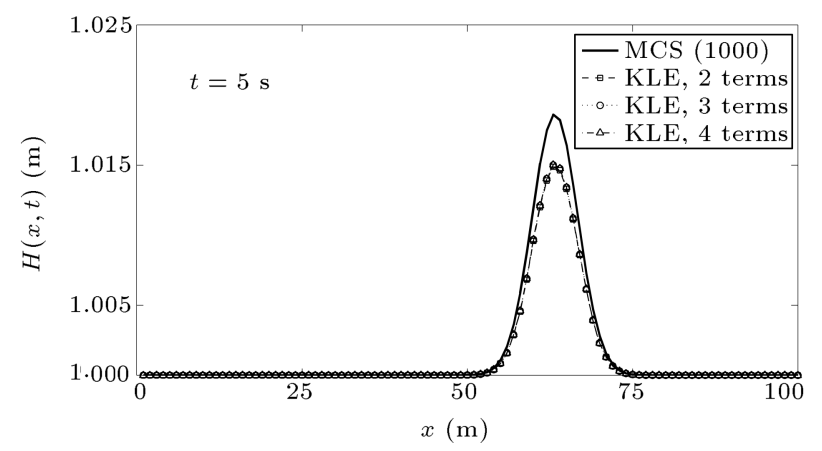

(a)

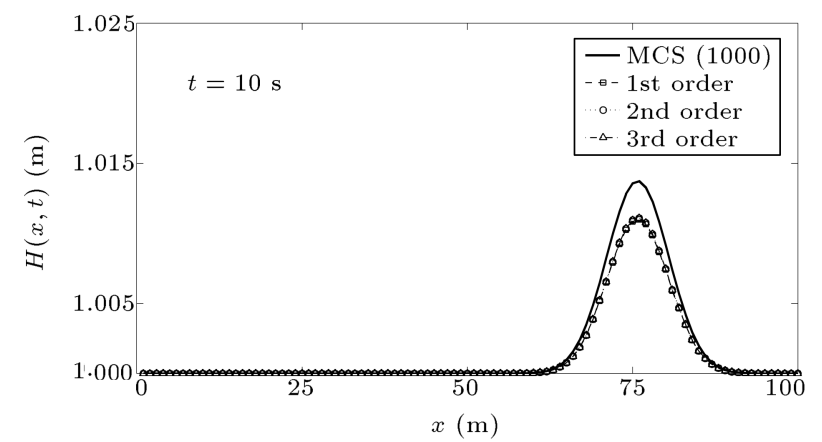

(b)

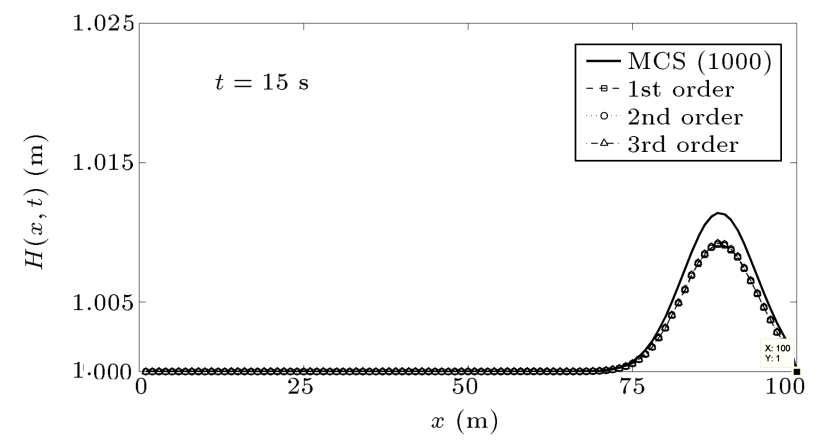

(c)

Figure 2. Comparison of mean flow depth profiles computed by KLE method (incorporating 2, 3, and 4 terms) and those derived by MCS (for 1000 realizations) at (a) $t=5 \mathrm{~s}$, (b) $t=10 \mathrm{~s}$, and (c) $t=15 \mathrm{~s}$. number of terms considered in its expansion. Moreover, $H^{(3)}(x, t)$ is obtained at the order of $0.0001 \mathrm{~m}$, i.e. two orders of magnitude smaller than $H^{(1)}(x, t)$. One may conclude that the more the number of terms incorporated in $H(x, t)$, the more accurate the results would be; however, incorporation of four terms $\left(H^{(0)}\right.$ to $\left.H^{(3)}\right)$ is deemed sufficient to expand $H(x, t)$, because of the size of the disturbance, $0.05 \mathrm{~m}$, caused by the solitary wave.

\subsection{Number of terms in expansion of $\boldsymbol{H}^{(m)}$}

Number of terms considered in expansion of $H^{(m)}$ has a direct effect on the accuracy of results in KLE approach. Figure 3 depicts magnitudes of $H_{i 1, i 2, \ldots, i m}^{(m)}$ for $m=1,2,3$ on the center of domain at $t=5 \mathrm{~s}$. The advantage of KLE largely depends on the number of terms required to approximate the $m$-order term $H^{(m)}$. On the other hand, the magnitude of $H^{(m)}$

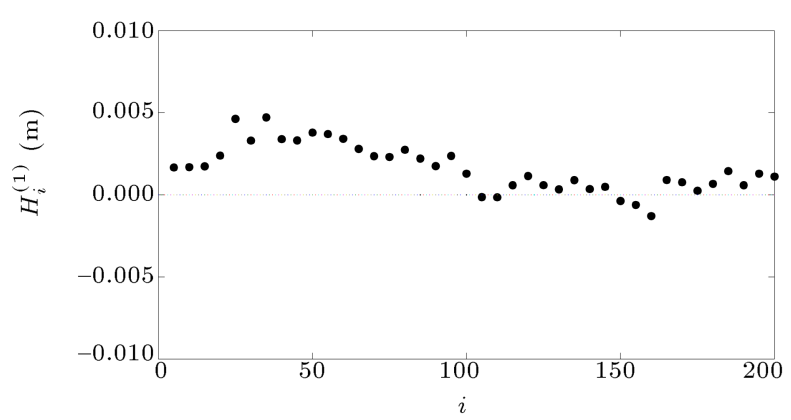

(a)

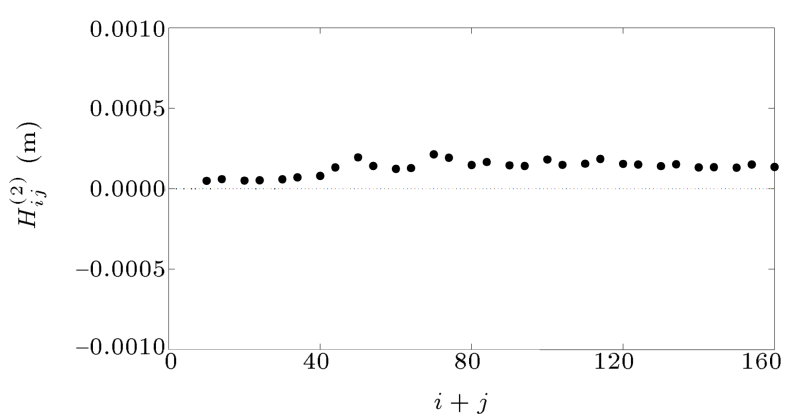

(b)

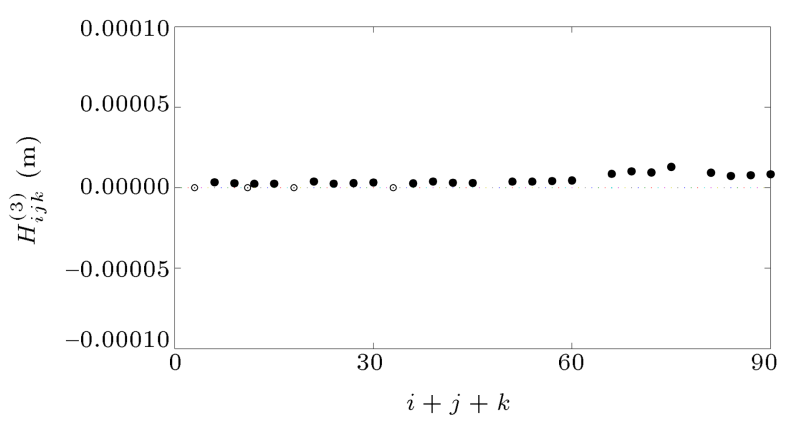

(c)

Figure 3. Values of $H_{i 1, i 2, \ldots, i m}^{(m)}$ on the center of domain at $t=5 \mathrm{~s}$ for $(\mathrm{a}) H_{i}^{(1)}$, (b) $H_{i}^{(2)}$, and (c) $H_{i j k}^{(3)}$. 
should statistically decrease for increasing values of the indices $i 1, i 2, \ldots, i m$. This condition is satisfied for $H^{(1)}$, although it seems that convergence is not achieved (cf. Figure 3(a) regarding the plot of $H^{(1)}$ for fixed $t=5 \mathrm{~s}$ ) when $H^{(2)}$ and $H^{(3)}$ show a non-decreasing behavior (cf. Figure 3(b) and (c) regarding the same time instant). In this computation, 100, 20, and 10 terms are found sufficient when evaluating $H_{i}^{(1)}, H_{i j}^{2}$, and $H_{i j k}^{3}$, respectively (Figure $3(\mathrm{a})$ to $(\mathrm{c})$ ), meaning that index $i$ in $H_{i}^{(1)}$ operates up to 100 , each index in $H_{i j}^{(2)}$ operates up to 20 , and so on. Number of times, $S_{m}$, necessary to solve governing equations for $H^{(1)}$ to $H^{(3)}$ is obtained from $S_{m}=\frac{n(n+1) \ldots(n+m-1)}{m !}$, where $n$ is the number of deterministic coefficients in any expansion procedure. When $N=15$, as an example, it is required to solve governing equations 120 and 680 times to compute $H^{(2)}$ and $H^{(3)}$, respectively; much less than the few thousand times usually needed for MCS.

\subsection{Dependency of flow depth variance $\left(\sigma_{H}^{2}\right)$ on input correlation length $\left(\eta_{h}\right)$}

Possible effects of input correlation length $\eta$ on flow depth variance, as one of the most important properties of the random function $H(x, t)$, are investigated. For this purpose, KLE approach is utilized to calculate the variance for different correlation lengths at $t=5 \mathrm{~s}$, which is later compared to MCS results (Figure 4(a) to (c)). As shown in figures, 1st and 2nd-order KLE yields almost identical results both revealing a fair agreement with MCS and overestimating the peak point by $12 \%$. Similar findings achieved for different correlation lengths suggest that flow depth variance is independent from input correlation length. This may be attributed to the fact that the roots of characteristic equation appear to be sensitive to correlation length.

\subsection{Effects of input variance $\left(\sigma_{h}^{2}\right)$ on flow depth variance $\left(\sigma_{H}^{2}\right)$}

Figure 5 compares flow depth variances from 1st and 2nd-order KLE approaches with those from MCS for different input water depth variances of $0.0025,0.0064$, and $0.0121 \mathrm{~m}^{2}$ and correlation length of 4 at $t=$ 5 s. As shown, for $\sigma_{h}^{2}=0.0025 \mathrm{~m}^{2}$, flow depth variances computed by 1 st and 2 nd-order KLE are close to the results of MCS with the 1st order mainly overestimating MCS with a maximum error of $9 \%$ (Figure 5(a)). As the variance increases, 1st-order KLE results overestimate MCS again with a maximum error of $9 \%$; however, 2nd-order KLE underestimates MCS with a maximum error of $23 \%$ (Figure $5(\mathrm{~b})$ ). Finally, for $\sigma_{h}^{2}=0.0121 \mathrm{~m}^{2}$, flow depth variances calculated by the 1st-order KLE remain unchanged (with errors similar to those in the previous cases), but high errors of up to $52 \%$ are observed for the underestimating 2 nd-

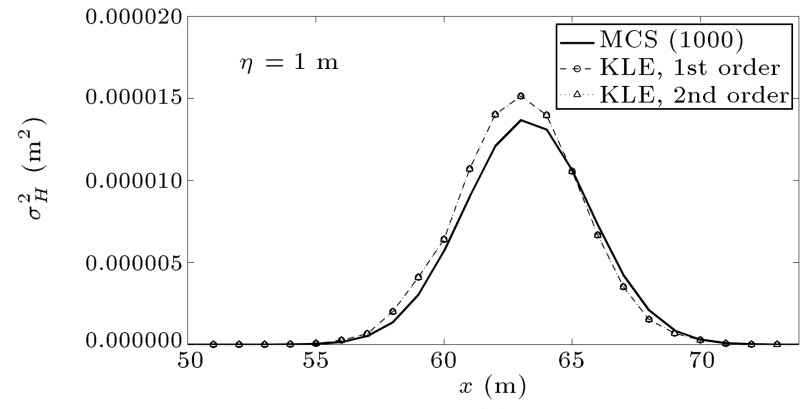

(a)

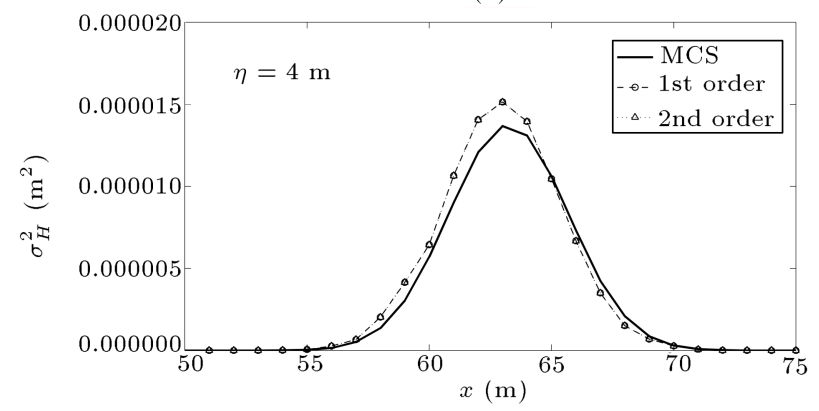

(b)

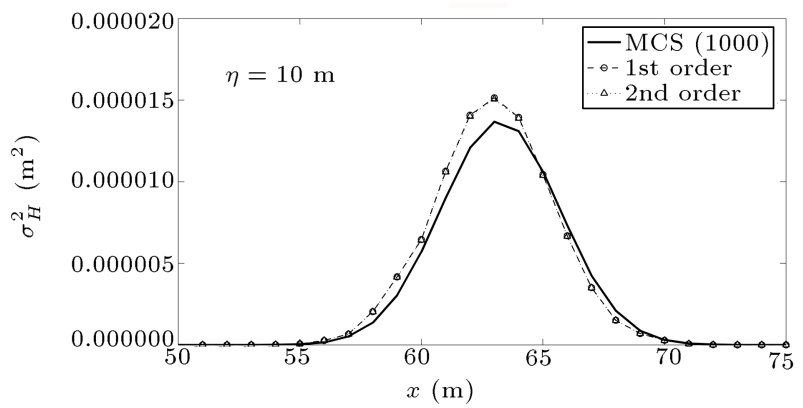

(c)

Figure 4. Comparison of flow depth variances computed by (first and second-order) KLE and MCS for: (a) $\eta_{h}=1 \mathrm{~m}$, (b) $\eta_{h}=4 \mathrm{~m}$, and (c) $\eta_{h}=10 \mathrm{~m}\left(\sigma_{h}^{2}=0.01 \mathrm{~m}^{2}\right.$ for all the cases).

order KLE (Figure 5(c)). It may be concluded that as the input variance, $\sigma_{h}^{2}$, increases, 1 st-order KLE results remain overestimated and unchanged; however, 2ndorder KLE results increasingly underestimate MCS results. It is concluded that for higher input variances, unlike our expectations, flow depth variance will not improve considerably by incorporation of higher order.

\section{Conclusions}

In this study, KLE approach was applied to solve stochastic ADE solution in a hypothetical channel considering uncertainties in the initial condition. Random variable, $h(x)$, was decomposed to infinite series based on eigenstructures of its covariance function. The latter was obtained applying Fredholm's solution to $1 \mathrm{D}$ exponential covariance function analytically. Then, flow depth $H(x, t)$ was expanded to series of $H^{(m)}$, each of which was further expanded to infinite 


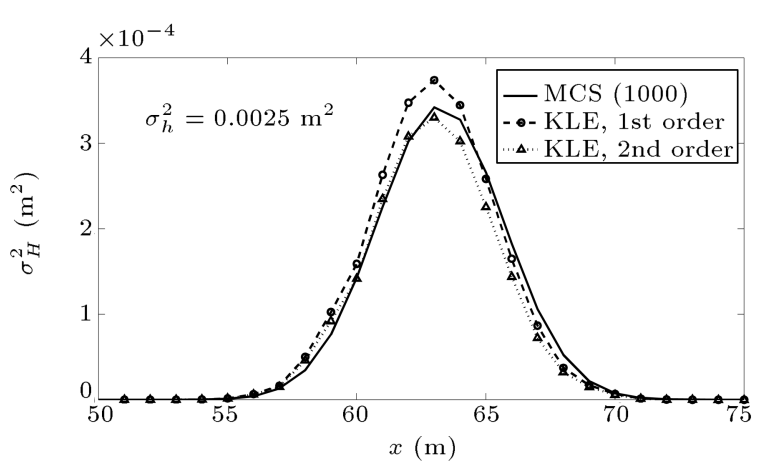

(a)

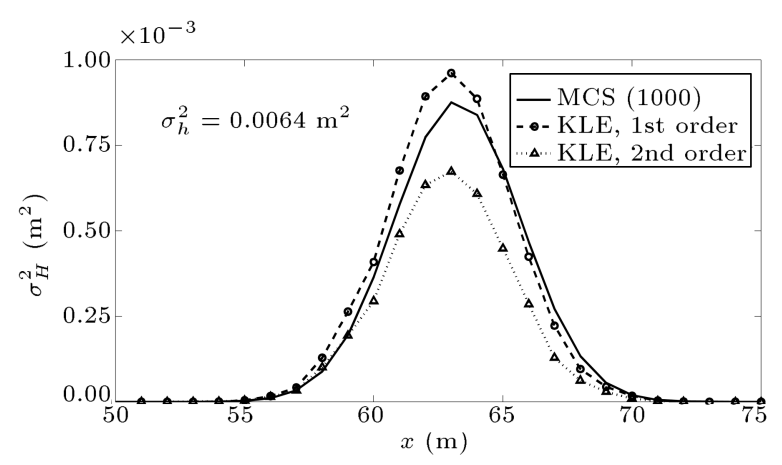

(b)

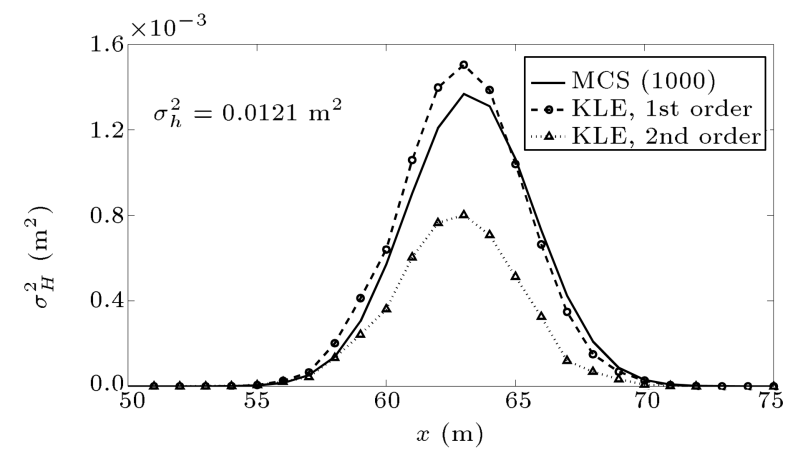

(c)

Figure 5. Comparison of flow depth variances computed by 1st and 2nd-order KLE with MCS, when (a) $\sigma_{h}^{2}=0.0025 \mathrm{~m}^{2}$, (b) $\sigma_{h}^{2}=0.0064 \mathrm{~m}^{2}$, and (c) $\sigma_{h}^{2}=0.0121 \mathrm{~m}^{2}\left(\eta_{h}=4 \mathrm{~m}\right.$ for all the cases $)$.

series based on random variable $\xi$. Coefficients of the last expansions were deterministically obtained from numerical solutions to the governing equation. A recursive method was utilized by KLE approach which, unlike PCE approach, did not require any coupling procedure to derive flow depth moments.

KLE approach was adopted to calculate mean flow depth profile at different times and compared with MCS results. It was concluded that the more the number of terms incorporated in $H(x, t)$, the more accurate the results would be; however, incorporation of four terms $\left(H^{(0)}\right.$ to $\left.H^{(3)}\right)$ was deemed sufficient for the size of disturbance $(0.05 \mathrm{~m})$ considered in this study. It was also found that 100,20 , and 10 terms were sufficient for computing $H_{i}^{(1)}, H_{i j}^{(2)}$, and $H_{i j k}^{(3)}$, respectively, resulting in much smaller number of times required to solve the governing equations than the few thousand times usually needed for MCS. Flow depth variance was found to be independent from input correlation length. Flow depth variance did not significantly improve when higher input variances were considered.

\section{References}

1. Ghanem, R.G. and Spanos, P.D. "Stochastic finite elements: a spectral approach", pp. 13-47, Springer, New York (2003).

2. Ghanem, R. "Scales of fluctuation and the propagation of uncertainty in random porous media", Water Resources Research, 34(9), pp. 2123-2136 (1998).

3. Ghanem, R. "Probabilistic characterization of transport in heterogeneous media", Computer Methods in Applied Mechanics and Engineering, 158(3), pp. 199220 (1998).

4. Xiu, D. and Karniadakis, G. E. "Modeling uncertainty in steady state diffusion problems via generalized polynomial chaos", Computer Methods in Applied Mechanics and Engineering, 191(43), pp. 4927-4948 (2002).

5. Sattar, A.M. "A probabilistic projection of the transient flow equations with random system parameters and internal boundary conditions", Journal of Hydraulic Research, pp. 1-18 (2016).

6. Ge, L., Cheung, K.F. and Kobayashi, M.H. "Stochastic solution for uncertainty propagation in nonlinear shallow-water equations", Journal of Hydraulic Engineering, 134(12), pp. 1732-1743 (2008).

7. Liu, D. "Uncertainty quantification with shallow water equations", Doctoral dissertation, University of Florence, Florence, Italy (2010).

8. Debusschere, B.J., Najm, H.N., Pébay, P.P., Knio, O.M., Ghanem, R.G. and Le Maĭtre, O.P. "Numerical challenges in the use of polynomial chaos representations for stochastic processes", SIAM Journal on Scientific Computing, 26(2), pp. 698-719 (2004).

9. Li, H. and Zhang, D. "Probabilistic collocation method for flow in porous media: Comparisons with other stochastic methods", Water Resources Research, 43(9), pp. 44-48 (2007).

10. Drakos, S. "Quantitative of uncertainty in unconfined flow problems", International Journal of Geotechnical Engineering, 10(3) pp. 213-222 (2016).

11. Kumar, Y.S., Li, J., Talarico, C. and Wang, J.A. "Probabilistic collocation method based statistical gate delay model considering process variations and multiple input switching", In Proceedings of the Conference on Design, Automation and Test in Europe, 2, pp. 770-775 (2005). 
12. Shi, L., Yang, J., Zhang, D. and Li, H. "Probabilistic collocation method for unconfined flow in heterogeneous media", Journal of Hydrology, 365(1), pp. 4-10 (2009).

13. Zhang, D. and Lu, Z. "An efficient, high-order perturbation approach for flow in random porous media via Karhunen-Loeve and polynomial expansions", Journal of Computational Physics, 194(2), pp. 773-794 (2004).

14. Erhel, J., Mghazli, Z. and Oumouni, M. "Numerical analysis of stochastic advection-diffusion equation via Karhunen-Loeve expansion", Research Report, p. 26 (2014).

15. Ueckermann, M.P., Lermusiaux, P.F.J. and Sapsis, T.P. "Numerical schemes for dynamically orthogonal equations of stochastic fluid and ocean flows", Journal of Computational Physics, 233, pp. 272-294 (2013).

16. Li, L., Tchelepi, H.A. and Zhang, D. "Perturbationbased moment equation approach for flow in heterogeneous porous media: applicability range and analysis of high-order terms", Journal of Computational Physics, 188(1), pp. 296-317 (2003).

17. Gates, T.K. and Al-Zahrani, M.A. "Spatiotemporal stochastic open-channel flow. I: Model and its parameter data", Journal of Hydraulic Engineering, 122(11), pp. 641-651 (1996).

18. Gates, T.K. and Al-Zahrani, M.A. "Spatiotemporal stochastic open-channel flow. II: Simulation experiments", Journal of Hydraulic Engineering, 122(11), pp. 652-661 (1996).

19. Liu, D.S. and Matthies, H.G. "Numerical treatment of shallow water equations with uncertain parameters", 8th.World Congress on Computational Mechanics (2008).

20. Dutykh, D., Katsaounis, T. and Mitsotakis, D. "Dispersive wave runup on non-uniform shores", In Finite Volumes for Complex Applications VI Problems and Perspectives, pp. 389-397 (2011).

21. Mohring, J., Milk, R., Ngo, A., Klein, O., Iliev, O., Ohlberger, M. and Bastian, P. "Uncertainty quantification for porous media flow using multilevel Monte Carlo", In Large-Scale Scientific Computing, 9374, pp. 145-152 (2015).

22. Mahdavi, A. and Talebbeydokhti, N. "Modeling of non-breaking and breaking solitary wave run-up using FORCE-MUSCL scheme", Journal of Hydraulic Research, 47(4), pp. 476-485 (2009).

23. Mahdavi, A., Reza Hashemi, M. and Talebbeydokhti, N. "A localized differential quadrature model for moving boundary shallow water flows", Journal of Hydraulic Research, 50(6), pp. 612-622 (2012).

24. Leonard, B.P. "A stable and accurate convective modelling procedure based on quadratic upstream interpolation", Comp. Meth. in Appl. Mech. and Engage., 19, pp. 59-98 (1979).
25. Abbott, M.B. and Basco, D.R. "Computational fluid dynamics", Harlow, Essex: Longman Scientific and Technical, New York, USA, pp. 146-151 (1989).

26. Szymkiewicz, R. "Numerical modeling in open channel hydraulics", 83, pp. 263-300, Springer Science and Business Media (2010).

\section{Appendix}

\section{Numerical approach}

i) Homogenous form. Homogeneous form of the governing equation (Table 1) was solved to obtain $H^{(0)}(x, t)$. Accordingly, QUICKEST (Quadratic Upstream Interpolation for Convective Kinematics with Estimated Streaming Terms) scheme, as the most frequently applied finite difference approach to solve ADE, was chosen [24-26]. QUICKEST utilizes up to the 3rd order of approximation and implements explicit centered differences as:

$$
\begin{aligned}
H_{i}^{j+1}= & H_{i}^{j}+\left[C_{d}\left(1-C_{a}\right)-\frac{C_{a}}{6}\left(C_{a}^{2}-3 C_{a}\right.\right. \\
& +2)] H_{i+1}^{j}-\left[C_{d}\left(2-3 C_{a}\right)\right. \\
& \left.-\frac{C_{a}}{2}\left(C_{a}^{2}-2 C_{a}-1\right)\right]+\left[C_{d}\left(1-3 C_{a}\right)\right. \\
& \left.-\frac{C_{a}}{2}\left(C_{a}^{2}-C_{a}-2\right)\right] H_{i-1}^{j}+\left[C_{d} \cdot C_{a}\right. \\
& \left.+\frac{C_{a}}{6}\left(C_{a}^{2}-1\right)\right] H_{i-2}^{j},
\end{aligned}
$$

in which $H_{i}^{j}$ is flow depth at the $i$ th $\Delta t$ (spatial step) and $j$ th $\Delta t$ (time step), and $C_{a}$ and $C_{d}$ are advective and diffusive Courant numbers, respectively:

$$
C_{a}=\frac{U \cdot \Delta t}{\Delta x}, \quad C_{d}=\frac{D \cdot \Delta t}{\Delta x^{2}} .
$$

Regions of stability for QUICKEST scheme may be written as [24]:

$$
\begin{cases}C_{d} \leq \frac{\left(3-2 C_{a}\right)\left(1-C_{a}^{2}\right)}{6\left(1-2 C_{a}\right)} & \text { if } C_{a}<\frac{1}{2} \\ C_{d} \geq \frac{\left(3-2 C_{a}\right)\left(C_{a}^{2}-1\right)}{6\left(2 C_{a}-1\right)} & \text { and } C_{d} \geq 0 \\ \text { if } C_{a}>\frac{1}{2}\end{cases}
$$

ii) Non-homogeneous form. The simplest form of ADE with a source term $f$ may be written as [26]:

$$
\frac{\partial H}{\partial t}+U \frac{\partial H}{\partial x}-D \frac{\partial^{2} H}{\partial x^{2}}-f=0
$$


A splitting technique is applied to decompose Eq. (A.4) in the form:

$$
\frac{\partial H}{\partial t}+F^{(1)}+F^{(2)}=0,
$$

where:

$$
F^{(1)}=U \frac{\partial H}{\partial x}-D \frac{\partial^{2} H}{\partial x^{2}} \quad \text { and } \quad F^{(2)}=-f .
$$

At any time step, $\Delta t$, Eq. (A.5) is solved in two stages. In the first stage:

$$
\begin{aligned}
& \frac{\partial H^{(1)}}{\partial t}+U \frac{\partial H^{(1)}}{\partial x}-D \frac{\partial^{2} H^{(1)}}{\partial x^{2}}=0, \\
& H^{(1)}(x, t)=H(x, t) .
\end{aligned}
$$

The QUICKEST scheme solves the homogeneous form of ADE as outlined earlier. The source term is taken into account via the second stage:

$$
\frac{\partial H^{(2)}}{\partial t}=f, \quad H^{(2)}(x, t)=H^{(1)}(x, t+\Delta t),
$$

subject to the results already obtained in the first stage as the initial condition. Finally, results of the second stage are considered as the values for new time steps $H(x, t+\Delta t) \sim H^{(2)}(x, t+\Delta t)$.

\section{Biographies}

Hossein Khorshidi received a BS degree in Civil Engineering from Yasouj Branch, Islamic Azad University, Yasouj, Iran, in 2004, and an MS degree in Hydraulic Structures, in 2008, from Shiraz University, Iran, where he is currently a PhD-degree student in the Department of Civil and Environmental Engineering. His research interests include stochastic modeling in open channel flows and free surface hydraulics.

Nasser Talebbeydokhti is a Professor of Hydraulic and Environmental Engineering in the Department of Civil and Environmental Engineering at Shiraz University, Shiraz, Iran, where he is currently Head of the Environmental Research and Sustainable Development Center. He has published more than 80 journal papers and 150 conference papers. His main areas of interest include hydraulic engineering, sediment transport, environmental engineering, and hydraulic structures.

Gholamreza Rakhshandehroo is a Professor of Civil Engineering in the Department of Civil and Environmental Engineering at Shiraz University, Shiraz, Iran, where he has published more than 40 journal papers and 100 conference papers. His main areas of interest include hydraulic engineering, groundwater mathematical modeling, environmental engineering, and climate change. 